
\title{
28 Research Suare \\ Co-culture with leukemia cells decreases proliferation and increases chemoprotective capacity of normal mesenchymal stromal cells
}

\section{Ruixia Hou}

The second hospital of shanxi medical university

\section{Wanfang Yang}

The second hospital of shanxi medical university

\section{Yaofang Zhang}

The second hospital of shanxi medical university

\section{Xiuhua Chen}

The second hospital of Shanxi medical university

\section{Fanggang Ren}

The second hospital of shanxi medical university

\section{Jing Xu}

The second hospital of Shanxi medical university

\section{Yanhong Tan}

The second hospital of shanxi medical university

\section{Zhifang Xu}

The second hospital of shanxi medical university

Jianmei Chang

The second medical of shanxi medical university

Hongwei Wang ( $\nabla$ wanghw68@hotmail.com )

The second Hospital of Shanxi Medical University https://orcid.org/0000-0002-2902-591X

\section{Research}

Keywords: mesenchymal stromal cells (MSCs), acute myeloid leukemia, cell migration, chemoresistance, apoptosis, hematopoietic microenvironment

Posted Date: March 5th, 2020

DOI: https://doi.org/10.21203/rs.3.rs-16127/v1

License: (c) (1) This work is licensed under a Creative Commons Attribution 4.0 International License. Read Full License 


\section{Abstract}

Background Bone marrow mesenchymal stromal cells (BM-MSCs) are essential structural and functional components of the BM microenvironment and play an important role in acute myeloid leukemia (AML) pathogenesis. BM-MSCs isolated from AML patients (AML-MSCs) show distinct signatures from normal BM-MSCs. However, the exact abnormalities of AML-MSCs and the origin of these abnormalities are still unknown.

Methods In this study, we evaluated the proliferative activity of AML-MSCs, and the influence of leukemia cells (LCs) on BM-MSCs. These two cell types were co-cultured using an in vitro co-culture system, and the biological functions of AML-MSCs, healthy donor derived MSCs (HD-MSCs), and LC-treated HD-MSCs (LCtrHD-MSCs) were compared by flow cytometry, and CCK-8 and chemotaxis assays. Student t-test (between two groups) and one-way ANOVA (more than 2 groups) were used to compare differences. Pearson correlation coefficients were used to assess correlations between two factors.

Results AML-MSCs display a significant proliferative deficiency, which correlates with primary leukemic blast cell counts but not with patients' age. Inhibition of BM-MSC proliferation could be induced by leukemia cells through direct contact. Co-cultured leukemia cells also increase expression of several inflammatory cytokines, and chemokines in BM-MSCs. Furthermore, LCtrHD-MSCs reduced apoptosis, and increased migration and chemoresistance in co-cultured AML cells, comparable with AML-MSCs.

Conclusions Our results showed that leukemia cells can induce healthy donor derived BM-MSCs to exhibit AML-MSC-like characteristics and indicated that AML-MSC abnormalities may be partly induced by leukemia cells.

\section{Background}

Acute myeloid leukemia (AML) is a heterogeneous clonal disorder marked by expansion and accumulation of immature myeloid blasts. Following advances in the understanding of the molecular and genetic alterations associated with $\mathrm{AML}$ and the development of chemotherapeutic agents, the majority of patients achieve complete remission (Rm) at initial treatment. However, patients eventually develop recurrence, which is very common and is partly due to the development of chemoresistance ${ }^{1}$. Multiple factors, for example, the existence of leukemic stem cells (LSCs) and the protective bone marrow (BM) niche have been found to be involved in the chemoresistance ${ }^{2,3}$. Although treatments targeting LSCs as well as the leukemia BM microenvironment have gained certain clinical effects, the interactions between leukemia cells (LCs) and their BM niche need further investigation.

BM derived mesenchymal stromal cells (BM-MSCs), also named as mesenchymal stem cells, are essential structural and functional components of the BM microenvironment and play an important role in hematopoiesis by regulating hematopoietic stem and progenitor cell (HSPC) quiescence, self-renewal, proliferation and differentiation ${ }^{4,5}$. In the past decades, many studies have demonstrated that BM-MSCs are important in the development of hematological malignancies and that they contribute to 
chemoresistance through the release of specific soluble cytokines ${ }^{6,7}$ or through their interaction with $\mathrm{LCs}^{8}$.

Therefore, despite advancements in our understanding of the molecular and genetic alterations in AML, the abnormalities in BM-MSCs derived from AML patients (AML-MSCs) have not been fully elucidated. AML-MSCs overexpress several chemokines and cytokines, such as stromal cell-derived factor-1 (SDF-1, also known as $\mathrm{CXCL} 12){ }^{9}$, prostaglandins (PGs) ${ }^{10}$, galectin $3{ }^{11}$, apoptosis repressor with caspase recruitment domain (ARC) protein ${ }^{12}$, and IL-10 ${ }^{13}$ and were more prefer to support LC survival compared with normal MSCs. Proteomic profiling has also revealed a different protein expression profile of AMLMSCs, and certain MSC protein expression signatures may be related to patient survival and remission ${ }^{11}$. Several studies have shown that abnormalities in AML-MSCs may contribute to AML pathogenesis 9,11 , 12 , but the origin of these abnormalities is still unclear. Cytogenetic analysis of tumor-specific genetic alterations has provided controversial results. Huang et al. have found that part of the AML patients carry mutations in their BM-MSCs, some of which overlap with and some that are distinct from their corresponding leukemic blasts ${ }^{13}$. However, another study performed on a larger cohort did not identify any molecular abnormalities in AML-MSCs ${ }^{14}$. Recent studies have demonstrated that LCs modify the normal hematopoietic niche into a "leukemia niche" to selectively support LC survival and induce a distinct cross-talk between LCs and MSCs ${ }^{15,16}$. However, whether the abnormalities in AML-MSCs originate from the influence of LCs or are due to mutations is still unclear. To evaluate the influence of LCs on MSCs, we cultured the two cell types using an in vitro co-culture system and compared the gene expression profile as well as the biological functions of the different MSCs.

\section{Methods}

\section{Patient samples}

BM was obtained from AML patients $(n=6$, age 18-70 years, average $=45.83 \pm 17.19)$ undergoing diagnostic BM aspiration. Individuals who were diagnosed as not having hematological diseases after BM aspiration were enrolled as healthy donors $(n=6$, age 20-65 years, average $=38.33 \pm 18.07)$. Informed consent was obtained from all participants in accordance with the guidelines of the Medical Ethics Committee of Shanxi Medical University (SXMU) and protocols involved in this study were approved by the Investigational Review Board of SXMU. Two $\mathrm{ml}$ of BM aspirate were collected from each participant and transferred into sodium citrate-containing tubes for MSC isolation. Patient characteristics are presented in Table 1.

\section{MSC isolation, culture and characterization}

Mononuclear cells were isolated from BM by Ficoll (specific gravity 1.077; Sigma-Aldrich, St. Louis, MO, USA) density gradient centrifugation. Cells were washed twice with PBS, resuspended at a concentration of $1.0 \times 10^{6}$ cells $/ \mathrm{mL}$ with DMEM/F12 containing $10 \%$ FBS and $1 \%$ antibiotic mixture (penicillin, 100 $\mathrm{U} / \mathrm{mL}$ and streptomycin sulphate, $100 \mu \mathrm{g} / \mathrm{mL}$ ) and then incubated at $37^{\circ} \mathrm{C}$ in T25 culture flasks in a 
humidified atmosphere supplemented with $5 \% \mathrm{CO}_{2}$. The non-adherent cells were removed after $48 \mathrm{~h}$ and the culture medium was changed every 3 days. Adherent MSCs were passaged at $80 \%$ confluency. Cell surface markers, such as CD105, CD29, CD44, CD45, CD34, and human leukocyte antigen (HLA)-DR (Becton Dickinson and Company, NY, USA) were detected using flow cytometry (Beckman Coulter, Inc., CA, USA). The MSCs were digested at passage 3 and reseeded in 24-well culture plates at a density of $1.0 \times$ $10^{5}$ cells $/ \mathrm{mL}$. Co-culture with primary LCs or LC lines were applied when MSCs reached $70 \%$ confluency. The AML patient derived (AML-), healthy donor derived (HD-), and LC treated HD (LCtrHD-) MSCs were used for comparison of their biological functions.

\section{Co-culture of LCs with MSCs}

Primary LCs from BM were separated using Ficoll gradient and seeded on a MSC layer in RPMI-1640 culture medium (Invitrogen, Carlsbad, CA, USA) supplemented with 10\% FBS (Gibco), 1\% penicillin/streptomycin at a concentration of $1 \times 10^{5}$ cells $/ \mathrm{mL}$.

Human AML cell line Dami (CRL9792-1071) was purchased from ATCC (American Type Culture Collection, Virginia, USA). Kasumi-1(SCSP-5015) and THP-1 (TCHu57) cell lines were purchased from Shanghai Cellular Institute of China Scientific Academy. UT-7, U937 and NB4 cells are curated in the Institute of Hematology, Second Hospital of of SXMU (Taiyuan, China). All cell lines were cultured according to specific culture guidelines. A concentration of $1 \times 10^{5} / \mathrm{mL}$ of AML cells was used to coculture with MSCs in a direct-contact or Transwell system.

\section{Assessment of cell survival, apoptosis, migration and drug resistance}

Viability of LCs cultured alone or co-cultured with MSCs was evaluated using a CCK-8 assay (Solarbio life science, Beijing, China). Briefly, cells were cultured in 96 -well plates under various culture conditions for $24-48 \mathrm{~h}$, then incubated with CCK-8 solution at $37^{\circ} \mathrm{C}$ for $2 \mathrm{~h}$. Absorbance was measured at $450 \mathrm{~nm}$. Cell viability was calculated according to manufacturer's guidelines. After co-culture for 3 days, AML cells were harvested and labeled with annexin-V (Becton Dickinson and Company, NY, USA) and cell apoptosis was analyzed by flow cytometry (Beckman Coulter, Inc., CA, USA). For chemotaxis assay, $1 \times 10^{5} \mathrm{HD}$-, AML- and LCtrHD-MSCs were seeded in the lower chamber of a 24 well Transwell culture plate (CorningCoster, NY, USA), and $1 \times 10^{6}$ THP-1 cells were added in the upper chamber of each well. Cells migrated into the lower chamber were counted after $24 \mathrm{~h}$. CCK-8 was used to evaluate the Arc-a resistance of THP1 cells cultured alone or in the co-presence of HD-, AML- or LCtrHD- MSCs.

\section{Detection of aldehyde dehydrogenase positive $\left(\mathrm{ALDH}^{+}\right)$LSCs}

To detect ALDH ${ }^{+}$leukemic stem cells, AML cell lines including NB4, U937, Dami, and Kasumi-1 were labeled with activated ALDEFLUOR ${ }^{\text {TM }}$ Reagent (ALDEFLUOR ${ }^{\text {TM }}$ Kit, StemCell, Vancouver, Canada) before and after co-culture with different MSCs. After incubation, flow cytometry was performed to detect the percent of $\mathrm{ALDH}^{+}$cells. 


\section{RNA sequencing}

The MSCs cultured alone or together with the AML cell line THP-1 were digested following washed thoroughly using PBS and $0.25 \%$ trypsin (if need) to remove THP-1 and collected for transcriptome sequencing. Briefly, total RNA was extracted using Trizol (Invitrogen, Carlsbad, CA, USA) and treated with DNase and a Ribo-Zero Magnetic Kit to digest double-stranded and single-stranded DNA and deplete rRNA, respectively. Purified mRNA was fragmented into small pieces and used to construct a cDNA library. Sequencing was performed on a BGISEQ500 platform (BGI, Shenzhen, China). The rRNA, low quality samples, linker contamination, and unknown base $\mathrm{N}$ content were filtered out from raw data. The clean reads were then mapped to the genome database and Bowtie2 were used to align the clean reads to the reference sequence. RSEM was used to calculate the number of reads mapping to genes. Differentially expressed genes between the 2 groups were screened using the criteria that log 2 fold change must be $\geq 1$ or $\leq-1$, $p$ value must be $\leq 0.001$, and the false discovery rate (FDR) must be $\leq$ 0.001 .

Data analysis

Data are presented as mean \pm standard error of the mean (SEM). SPSS ** software (Math Soft, Inc., Seattle, WA, USA) was used to perform statistical analyses. Student t-test (between two groups) and one-way ANOVA (more than 2 groups) were used to compare differences. The Pearson correlation was used to evaluate correlations between two factors.

\section{Results}

\section{AML-MSCs showed reduced proliferative capacity}

We expanded AML-MSCs $(n=6)$ and HD-MSCs $(n=6)$ and evaluated the phenotypic and biological characteristics of both cell groups. Both AML-MSCs and HD-MSCs displayed similar spindle-shaped fibroblast like morphologies (Fig. 1a), expressed typical MSC markers $\left(\mathrm{CD} 105^{+} \mathrm{CD} 29^{+} \mathrm{CD} 44^{+}\right)$and were negative for hematopoietic markers (CD45 $\left.{ }^{-} \mathrm{CD} 34^{-} \mathrm{HLA}^{-D R^{-}}\right)$in a comparable way (Supplementary Fig. 1). During MSC expansion, we found that AML-MSCs showed decreased proliferative activity. The adherent cells in primary AML-MSC cultures were significantly less than those in HD-MSCs cultures at the same culture time (Fig. 1a). AML-MSCs needed 22-37 days to reach $80 \%$ confluency, while HD-MSCs needed $12-20$ days (average $=29.17 \pm 6.11$ vs. $16.00 \pm 3.63, p<0.01$, Fig. $1 b$ ). This difference declined with prolonging the culture time in vitro. When cultured at passage 3 , no significant differences were found between the two groups ( $7.83 \pm 1.33$ vs. $6.83 \pm 0.75, p>0.05$, Fig. $1 b)$. The proliferative activity of MSCs may be influenced by various factors such as patients' age, leukemic blast cell counts etc. Thus, we carried out a correlative analysis between these factors and the confluency time of primary culture. As shown in Fig. 1c and 1d, there was a significant positive correlation between the primary leukemic blast cell count and confluency time (Pearson $r=0.8494, p=0.0323$, Fig. $1 c$ ). In contrast, no correlation was 
found with patients' age (Pearson $r=0.5022, p=0.0962$, Fig. 1d). These results indicate that the decreased proliferative activity may be induced by AML cells but is not related to patients' age.

\section{LCs suppress MSC proliferation via direct cell contact}

To further evaluate the influence of LCs on MSC proliferation, we co-cultured AML cell lines THP-1 and UT7 with HD-MSCs in three different ways: $\triangle$ direct-contact (DC): AML cell lines were seeded on the HD-MSC

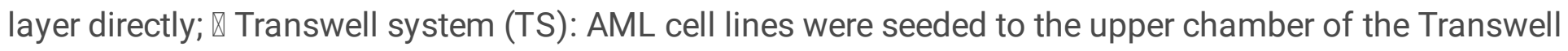
insert; $₫$ conditioned culture medium (CM): the culture supernatants of AML cell lines were added to the HD-MSC culture system. After co-culture for 3 days, the LCs were removed and the MSCs were digested for cell counting. As shown in Fig. 2a-c, the MSC cell numbers were comparable in MSCs cultured alone (W/O), in TS, and in CM, but were significantly reduced in DC. Thus, neither TS nor CM inhibited the proliferation of HD-MSCs, but the LCs that contacted with HD-MSCs directly inhibited their proliferation. This result is consistent with the results of other studies, which have reported that AML-MSCs demonstrated reduced proliferation rate compared to control MSCs ${ }^{17,18}$, and confirmed that the decreased proliferative potential of AML-MSCs was induced, at least partly, by LCs via cell-cell contact.

\section{LCs change the MSC gene and cytokine expression}

In addition to the proliferative activity, the effect of LCs on the biological characteristics of co-cultured MSCs needs further investigation. Increased expression of CCL2, CCL4, CXCL12, IL-8, PGE2, leukemia inhibitory factor (LIF), and IL-6 has been reported in AML-MSCs as well as MSCs isolated from other solid carcinomas ${ }^{19-21}$. Here, we analyzed IL-6, IL-8, macrophage migration inhibitory factor (MIF), and LIF expression in AML-MSC cultures and HD-MSC cultures by ELISA. As shown in Fig. 3a, IL-6, IL-8 and MIF were high expressed in AML-MSCs compared to HD-MSCs. To further investigate whether the abnormal of AML-MSCs were induced by LCs, we co-cultured HD-MSCs with AML cell line THP-1, and then compare the gene expression between HD-MSCs cultured alone and co-cultured with THP-1. Transcriptome sequencing analysis showed a significantly different gene expression profile between the two cell groups (Fig. 3b). Sequencing data were deposited in the China National Gene Bank (CNGB, CNP0000695). There were totally 2808 differently expressed genes (2182 up regulated and 626 down regulated) between the two cell groups (Fig. 3c). The differentially expressed genes were mainly enriched in cytokine-cytokine receptor interaction, pathways in cancer, calcium signaling pathway, transcriptional mis-regulation in cancer, chemokine signaling pathway, osteoclast differentiation, leukocyte trans endothelial migration etc. Genes which involved in cell cycle were also influenced by THP-1 co-culture. (Fig. 3d). It is noteworthy that, the expression of chemokines as well as inflammatory cytokines such as CCL3, CCL5, CCL23, CCL3L3, IL-18, IL-32, IL-34, TNF, IL-23A and their receptors IL2RG, IL-6R, IL-15RA, IL-27RA, IL-10RA, and CXCR4 was significantly up-regulated after co-culture with LCs (Fig. 3e).

\section{BM-MSCs co-culture did not increase the number of ALDH + LSCs}

ALDH is increasingly regarded as a leukemia-initiating cell (LIC) or LSC marker ${ }^{22}$. ALDH + LCs are more quiescent, exhibit higher cell proliferation rates, higher clonal efficiency, and increased chemotherapy resistance $23-25$. It has been reported that co-culture of colorectal carcinoma cells with MSCs results in a 
significant increase in ALDH1 protein levels and $\mathrm{ALDH}^{+}$populations compared with carcinoma cells cultured alone; co-culture with MSCs can promote entrance of carcinoma cells into a stem-cell-like state through PGE2 and other cytokines secreted by $\mathrm{MSCs}^{21}$. However, whether this occurs in co-cultures of LCs with bone marrow MSCs is still unknown. To clarify this issue, we co-cultured the LC lines NB4, Dami, Kasumi-1, or U937 with HD-MSCs or AML-MSCs. Among the four LC lines, NB4, Dami and U937 expressed certain levels of ALDH1, while in Kasumi- $1, \mathrm{ALDH}^{+}{ }^{+}$cells are almost undetectable (Fig. 4). After coculture, neither HD-MSCs nor AML-MSCs increased the percentage of ALDH1 ${ }^{+}$LCs (Fig. 4, p >0.05). This result suggests that, at least in AML LCs, co-culture with MSCs is unable to enhance the number of $\mathrm{ALDH} 1^{+}$LSCs.

\section{LC-treated MSCs support survival and promote migration of AML cells}

The BM niche supports both normal CD34 + HSPC and AML cell survival and proliferation, and the AMLMSCs have been reported to support LC survival and proliferation ${ }^{15-17,19}$. To study the influence of different MSCs on AML cell viability, apoptosis, migration, and drug resistance, we co-cultured THP-1 cells together with HD-MSCs, AML-MSCs, or LCtrHD-MSCs. We showed a significant difference in THP-1 apoptosis, migration, and drug resistance between the different cultures. All the three kinds of MSCs supported cell viability similarly in vitro, although the AML- and LCtrHD-MSCs slightly increased the viability of co-cultured THP-1 cells, but the difference was not significant $(115.77 \pm 13.26 \%$ and $110.82 \pm$ $33.04 \%$ respectively vs. $100 \%, p>0.05$. Table 2 and Fig. $5 a)$. All the three kinds of MSCs reduced the apoptosis rate of co-cultured THP-1 cells. Both AML- and LCtrHD-MSCs showed stronger anti-apoptosis ability than HD-MSCs (Table 2 and Fig. 5b,c), but the difference between AML- and LCtrHD-MSCs was not significant ( $3.35 \pm 1.11 \%$ vs. $4.32 \pm 1.42 \%, p>0.05)$. Regarding chemotaxis, both AML- and LCtrHD-MSCs attracted more LCs than HD-MSCs $\left(2.76 \pm 0.47 \times 10^{5}\right.$ and $1.92 \pm 0.26 \times 10^{5}$ respectively vs. $0.66 \pm 0.25 \times$ $10^{5}, p<0.05$. Table 2 and Fig. $5 d$ ), indicating that co-culture with LCs can increase the chemotaxis of MSCs. To evaluate the chemoresistance, THP-1 cells were co-cultured for $48 \mathrm{~h}$ with different MSCs in the presence or absence of Arc-a and cell viability was examined by using the CCK-8 assay. As shown in supplementary Fig. 2a-d, Arc-a did not significantly inhibit BM-MSCs viability even at a concentration of $4 \mathrm{mM}$, while the $\mathrm{IC}_{50}$ of Arc-a on THP-1 cells was $1.2 \mathrm{mM}$ at $48 \mathrm{~h}$. Figure $5 \mathrm{e}$ shows the influence of different MSCs on THP-1 chemoresistance. Compared to THP-1 cells cultured alone, HD-MSCs did not improve chemoresistance ( $34.99 \pm 3.61 \%$ vs. $45.64 \pm 6.82 \%, p>0.05$. Table 2$)$, while the AML- and LCtrHDMSCs significantly increased the percent of Arc-a resistant THP- 1 cells $(67.41 \pm 5.82 \%$ and $59.49 \pm$ $11.00 \%$ respectively vs. $45.64 \pm 6.82 \%, p<0.05$. Table 2 ). AML-MSCs and LCtrHD-MSCs are more effective in reducing drug resistance.

\section{Discussion}

BM-MSCs have been used to reduce graft-versus-host disease (GVHD) because of their immunomodulatory effects. Normal BM-MSCs have been shown to inhibit the proliferation of LCs and 
increase peripheral $\mathrm{CD} 3^{+} \mathrm{CD} 8^{+} \mathrm{T}$ cells fraction ${ }^{26}$. Furthermore co-transplantation of MSCs has been shown to improve patient outcome after allogeneic hematopoietic stem cell transplantation (alloHSCT $)^{27}$. However, an increasing number of studies in recent years have reported that BM-MSCs contribute to the pathogenesis and development of myelogenous leukemias, including $A M L$, chronic myelogenous leukemia (CML), and myelodysplastic syndrome (MDS) $8,9-11,14,16,17$. BM-MSCs isolated from leukemia patients may have different biological characteristics compared to those from healthy individuals.

In this study, we found that AML-MSCs showed decreased proliferative potential in primary culture, which was related with the leukemic blast cell counts but not the age of patients. Certainly, the longer confluence time of AML-MSCs primary culture may partly attributed to the lower number of plated mesenchymal progenitors in the primary culture, which reflects an AML related MSCs intrinsic defect or an in vivo inhibition of LCs on bone marrow MSCs. Our results also revealed that the proliferative capacity of AML-MSCs were recovered when cultured at passage 3 , which indicating that the decreased proliferation of AML-MSCs may originated from LCs inhibition. In vitro co-culture also confirmed the ability of LCs to suppress proliferation of BM-MSCs. Corradi et al. have found approximately $25 \%$ of AMLMSCs failed to expand during in vitro culture, while MSCs obtained from HDs and MDS patients could be easily established, indicating that AML-MSCs demonstrated a reduced proliferation potential ${ }^{18}$. Furthermore, reduced proliferation capacity and increased adipogenic potential of AML-MSCs has also reported by other researchers ${ }^{28,29}$. When human LCs were transplanted into immuno-deficient mice, the BM microenvironments (including vascular structures and osteoblastic-lining cells) were damaged along with the leukemic dissemination, and subsequent chemotherapy could induce the formation of a protective niche around the residual $\mathrm{LCs}^{30}$. This research suggested that LCs can also inhibit proliferation and function of niche cells in vivo. In addition, AML-MSCs and MDS-MSCs have been shown to exhibit a senescence-associated phenotype and function, including a large flat morphology, increased $\beta$ galactosidase activity, and increased secretion of pro-inflammatory cytokines as well as chemokines ${ }^{31}$, 32. We hypothesized that, both the decreased proliferative capacity and the senescence-associated phenotype are probably induced by leukemic blasts.

Studies using leukemic mice models have reported that LCs transplanted into a mouse model can "hijack" the normal BM niche into a leukemic niche which impairs normal hematopoiesis, favors leukemic stem cell survival and expansion ${ }^{15}, 33$. It has been shown that AML-MSCs show special characteristics including over expression of a series of chemokines and cytokines ${ }^{11-13,31,32}$, increased adipogenic and osteoblastic potential, and improved the ability to support leukemia progenitor cells survival ${ }^{29,34}$. However, there are also opposite reports. Diaz et al. have compared the function, genetic and immunological characteristics of HD- and AML-MSCs, and found that the two MSCs protected AML cells equally towards Arc-a in vitro, while AML-MSCs showed enhanced suppression of lymphocyte proliferation, higher clonogenic potential, and diminished secretion of pro-inflammatory cytokines ${ }^{14}$. Nevertheless, despite the controversial findings, it is commonly accepted that AML-MSCs have different gene/protein expression signatures and were related to patient outcome. However, the origin of these 
abnormalities is still unclear. How MSCs are influenced by LCs still needs further investigation. To clarify this issue, we co-cultured the AML cell line THP-1 with HD-MSCs or AML-MSCs, and compared the gene expression profile and biological function between co-cultured HD-MSCs and AML-MSCs. We found that, co-culture with THP-1 increased the expression of some chemokines as well as inflammatory cytokines in MSCs, and THP-1 co-cultured HD-MSCs showed similar anti-apoptosis, chemoprotection and chemotaxis capacity as AML-MSCs. Our results indicating that LCs can induce MSCs to exhibit AML-MSCs like characteristics. But, neither HD-MSCs nor AML-MSCs co-cultures increased the percentage of ALDH1 ${ }^{+}$ LCs. Therefore, co-culturing with MSCs did not induce a stem cell like phenotype in LCs. This result differs from that using solid carcinoma derived MSCs, which may be because of the different crosstalk between different MSCs and tumor cells.

\section{Conclusions}

In conclusion, we investigated the influence of LCs on MSCs and confirmed that LCs can induce HDMSCs to exhibit an AML-MSCs like phenotype. Therefore, the aberrant characteristics of AML-MSCs may be induced at least partly by LCs. However, considering the heterogeneity of AML, it is necessary to conduct additional studies to further evaluate the influence of the other cell lines on MSCs and their exact mechanisms.

\section{Declarations}

- Ethics approval and participant consent: Informed consent was obtained from all participants in accordance with the guidelines of the Medical Ethics Committee of Shanxi Medical University (SXMU). Protocols used in this study were approved by the Investigational Review Board of SXMU (Approval number 2016083).

- Consent for publication: Publication of this manuscript has been approved by all co-authors.

- Availability of data and materials: We will make data and materials described in this manuscript freely available to scientists wishing to use them for non-commercial purposes.

- Competing interests: All authors declare no conflicts of interest.

- Funding: This work was supported by National Natural Science Foundation of China (N0.81670126 and 81602768), the Natural Science Foundation of Shanxi Province (NO. 201801 D111003 and 201801D221441), and the graduate educational innovation projects of Shanxi Province (No. 2016BY083).

- Authors' contributions: Wang Hongwei: Conceptualization, Visualization, Funding acquisition and Writing-review \& editing. Hou Ruixia: Conceptualization, Investigation, and Writing-original draft. Yang Wanfang: Investigation, Data curation, Project administration; Zhang Yaofang and Ren Fanggang: Methodology, Data curation, and Supervision. Chen Xiuhua: Resources, Methodology,and Formal analysis. Xu Jing and Chang Jianmei: Software and Validation. Tan Yanhong: Supervision and Writing - review \& editing. Xu Zhifang: Conceptualization and Formal analysis. 
- Acknowledgements: The authors acknowledge nurse Chenhua Yin for her great help with sample collection. We also thank all the patients for their voluntary participation in this study.

\section{References}

1. Dohner H, Estey E, Grimwade D, Amadori S, Appelbaum FR, Buchner T, Dombret H, Ebert BL, Fenaux P, Larson RA, Levine RL, Lo-Coco F, Naoe T, Niederwieser D, Ossenkoppele GJ, Sanz M, Sierra J, Tallman MS, Tien HF, Wei AH, Löwenberg B, Bloomfield CD. Diagnosis and management of AML in adults: 2017 ELN recommendations from an international expert panel. Blood. 2017;129:424-47.

2. Li H, Li J, Cheng J, Chen X, Zhou L, Li Z. AML-derived mesenchymal stem cells upregulate CTGF expression through the BMP pathway and induce K562-ADM fusiform transformation and chemoresistance. Oncol Rep. 2019; 42(3):1035-1046.

3. Carter BZ, Mak PY, Wang X, Tao W, Ruvolo V, Mak D, Mu H, Burks JK, Andreeff M. An ARC-Regulated IL1 $\beta /$ Cox-2/PGE2/ $\beta$-Catenin/ARC Circuit Controls Leukemia-MicroenvironmentInteractions and Confers Drug Resistance in AML. Cancer Res. 2019;79(6):1165-1177

4. Méndez-Ferrer S, Michurina TV, Ferraro F, Mazloom AR, Macarthur BD, Lira SA, Scadden DT, Ma'ayan A, Enikolopov GN, Frenette PS. Mesenchymal and haematopoietic stem cells form a unique bone marrow niche. Nature. 2010;466 (7308):829-834.

5. Morrison SJ, Scadden DT. The bone marrow niche for haematopoietic stem cells. Nature. 2014;505(7483):327-334.

6. Brenner AK, Nepstad I, Bruserud Ø. Mesenchymal stem cells support survival and proliferation of primary human acute myeloid leukemia cells through heterogeneous molecular mechanisms. Front Immunol. 2017;8: doi: 10.3389/ fimmu.2017.00106.

7. Reikvam H, Hatfield KJ, Fredly H, Nepstad I, Mosevoll KA, Bruserud O. The angioregulatory cytokine network in human acute myeloid leukemia - from leukemogenesis via remission induction to stem cell transplantation. Eur Cytokine Netw. 2012;23:140-153.

8. Zhang B, Li M, McDonald T, Holyoake TL, Moon R.T, Campana D, Shultz L, Bhatia R. Microenvironmental protection of $\mathrm{CML}$ stem and progenitor cells from tyrosine kinase inhibitors through N-cadherin and Wnt- $\beta$-catenin signaling. Blood. 2013;121:1824-1838.

9. Abe-Suzuki S, Kurata M, Abe S, Onishi I, Kirimura S, Nashimoto M, Murayama T, Hidaka M, Kitagawa M. CXCL12+ stromal cells as bone marrow niche for CD34+ hematopoietic cells and their association with disease progression in myelodysplastic syndromes. Lab Invest.2014;94(11):12121223.

10. Wu L, Amarachintha S, Xu J, Oley F Jr, Du W. Mesenchymal COX2-PG secretome engages NR4A-WNT signalling axis in haematopoietic progenitors to suppress anti-leukaemia immunity. $\mathrm{Br} \mathrm{J}$ Haematol. 2018;183(3):445-456.

11. Kornblau SM, Ruvolo PP, Wang RY, Battula VL, Shpall EJ, Ruvolo VR, McQueen T, Qui Y, Zeng Z, Pierce S, Jacamo R, Yoo SY, Le PM, Sun J, Hail N Jr, Konopleva M, Andreeff M. Distinct protein 
signatures of acute myeloid leukemia bone marrow-derived stromal cells are prognostic for patient survival. 2018;103(5):810-821.

12. Carter BZ, Qiu YH, Zhang N, Coombes KR, Mak DH, Thomas DA, Ravandi F, Kantarjian HM, Koller E, Andreeff M, Kornblau SM. Expression of ARC (apoptosis repressor with caspase recruitment domain), an antiapoptotic protein, is strongly prognostic in AML. Blood. 2011;117:780-787.

13. Huang JC, Basu SK, Zhao X, Chien S, Fang M, Oehler VG, Appelbaum FR, and Becker PS. Mesenchymal stromal cells derived from acute myeloid leukemia bone marrow exhibit aberrant cytogenetics and cytokine elaboration. Blood Cancer J. 2015;5(4): e302.

14. Diaz de la Guardia R, Lopez-Millan B, Lavoie JR, Bueno C, Castaño J, Gómez- Casares M, Vives S, Palomo L, Juan M, Delgado J, Blanco ML, Nomdedeu J, Chaparro A, Fuster JL, Anguita E, RosuMyles M, Menéndez P. Detailed characterization of mesenchymal stem/stromal cells from a large cohort of AML patients demonstrates a definitive link to treatment outcomes. Stem Cell Reports.2017;8(6):1573-1586.

15. Schepers K, Pietras EM, Reynaud D, Flach J, Binnewies M, Garg T, Wagers AJ, Hsiao EC, Passegué E. Myeloproliferative neoplasia remodels the endosteal bone marrow niche into a self-reinforcing leukemic niche. Cell Stem Cell.2013;13(3):285-299.

16. Kim JA, Shim JS, Lee GY, Yim HW, Kim TM, Kim M, Leem SH, Lee JW, Min CK, Oh IH. Microenvironmental remodeling as a parameter and prognostic factor of heterogeneous leukemogenesis in acute myelogenous leukemia. Cancer Res.2015;75(11):2222-2231.

17. Yehudai-Resheff S, Attias-Turgeman S, Sabbah R, Gabay T, Musallam R, Fridman-Dror A, Zuckerman T. Abnormal morphological and functional nature of bone marrow stromal cells provides preferential support for survival of acute myeloid leukemia cells. Int J Cancer.2019;144(9):2279-2289.

18. Corradi G, Baldazzi C, Očadlíková D, Marconi G, Parisi S, Testoni N, Finelli C, Cavo M, Curti A, Ciciarello M. Mesenchymal stromal cells from myelodysplastic and acute myeloid leukemia patients display in vitro reduced proliferative potential and similar capacity to support leukemia cell survival. Stem Cell Res Ther.2018;9(1):271.

19. Carter BZ, Mak PY, Chen Y, Mak DH, Mu H, Jacamo R, Ruvolo V, Arold ST, Ladbury JE, Burks JK, Kornblau S, Andreeff M. Anti-apoptotic ARC protein confers chemoresistance by controlling leukemia-microenvironment interactions through a NFKB/IL1 $\beta$ signaling network. 2016;7(15):2005420067.

20. Konoplev S, Rassidakis GZ, Estey E, Kantarjian H, Liakou Cl, Huang X, Xiao L, Andreeff M, Konopleva M, Medeiros LJ. Overexpression of CXCR4 predicts adverse overall and event-free survival in patients with unmutated FLT3 acute myeloid leukemia with normal karyotype. Cancer. 2007;109:1152-1156.

21. Li HJ, Reinhardt F, Herschman HR, Weinberg RA. Cancer-stimulated mesenchymal stem cells create a carcinoma stem cell niche via prostaglandin E2 signaling. Cancer Discov.2012;2(9):840-

22. Blume R, Rempel E, Manta L, Saeed BR, Wang W, Raffel S, Ermakova O, Eckstein V, Benes V, Trumpp $A, H o A D$, Lutz $C$. The molecular signature of AML with increased ALDH activity suggests a stem cell origin. Leuk Lymphoma. 2018;59(9):2201- 
23. Bogen A, Buske C, Hiddemann W, Bohlander SK, Christ O. Variable aldehyde dehydrogenase activity and effects on chemosensitivity of primitive human leukemic cells. Exp Hematol.2017;47:54-

24. Hoang VT, Buss EC, Wang W, Hoffmann I, Raffel S, Zepeda-Moreno A, Baran N, Wuchter P, Eckstein V, Trumpp A, Jauch A, Ho AD, Lutz C. The rarity of ALDH(+) cells is the key to separation of normal versus leukemia stem cells by ALDH activity in AML patients. Int J Cancer.2015;137(3):525-

25. Moreb JS, Ucar D, Han S, Amory JK, Goldstein AS, Ostmark B, Chang LJ. The enzymatic activity of human aldehyde dehydrogenases $1 \mathrm{~A} 2$ and 2 (ALDH1A2 and ALDH2) is detected by Aldefluor, inhibited by diethylaminobenzaldehyde and has significant effects on cell proliferation and drug resistance. Chem Biol Interact. 2012;195(1):52-

26. Song N, Gao L, Qiu H, Huang C, Cheng H, Zhou H, Lv S, Chen L, Wang J. Mouse bone marrow-derived mesenchymal stem cells inhibit leukemia/ lymphoma cell proliferation in vitro and in a mouse model of allogeneic bone marrow transplant. Int J Mol Med.2015;36(1):139-

27. Liu X, Wu M, Peng Y, Chen X, Sun J, Huang F, Fan Z, Zhou H, Wu X, Yu G, Zhang X, Li Y, Xiao Y, Song C, Xiang AP, Liu Q. Improvement in poor graft function after allogeneic hematopoietic stem cell transplantation upon administration of mesenchymal stem cells from third-party donors: a pilot prospective study. Cell Transplant. 2014;23(9):1087-

28. Desbourdes L, Javary J, Charbonnier T, Ishac N, Bourgeais J, Iltis A, Chomel JC, Turhan A, Guilloton F, Tarte K, Demattei MV, Ducrocq E, Rouleux-Bonnin F, Gyan E, Hérault O, Domenech J. Alteration Analysis of Bone Marrow Mesenchymal Stromal Cells from De Novo Acute Myeloid Leukemia Patients at Diagnosis. Stem Cells Dev. 2017;26(10):709-

29. Azadniv M, Myers JR, McMurray HR, Guo N, Rock P, Coppage ML, Ashton J, Becker MW, Calvi LM, Liesveld JL. Bone marrow mesenchymal stromal cells from acute myelogenous leukemia patients demonstrate adipogenic differentiation propensity with implications for leukemia cell support. 2019. doi: 10.1038/s41375-019-0568-8. [Epub ahead of print]

30. Duan CW, Shi J, Chen J, Wang B, Yu YH, Qin X, Zhou XC, Cai YJ, Li ZQ, Zhang F, Yin MZ, Tao Y, Mi JQ, Li LH, Enver T, Chen GQ, Hong DL. Leukemia propagating cells rebuild an evolving niche in response to therapy. Cancer Cell. 2014;25(6): 778-793.

31. Mattiucci D, Maurizi G, Leoni P, Poloni A. Aging- and senescence-associated changes of mesenchymal stromal cells in myelodysplastic syndromes. Cell Transplant.2018;27(5):754-

32. Abdul-Aziz AM, Sun Y, Hellmich C, Marlein CR, Mistry J, Forde E, Piddock RE, Shafat MS, Morfakis A, Mehta T, Palma FD, Macaulay I, Ingham CJ, Haestier A, Collins A, Campisi J, Bowles KM, Rushworth SA. Acute myeloid leukemia induces pro-tumoral p16INK4a driven senescence in the bone marrow microenvironment. Blood. 2018, Online published. DOI: 10.1182/blood-2018-04 -845420.

33. Zhang B, Ho YW, Huang Q, Maeda T, Lin A, Lee SU, et al. Altered microenvironmental regulation of leukemic and normal stem cells in chronic myelogenous leukemia. Cancer Cell. 2012;21:577-592.

34. Li H, Li J, Cheng J, Chen X, Zhou L, Li Z. AML-derived mesenchymal stem cells up-egulate CTGF expression through the BMP pathway and induce K562-ADM fusiform transformation and chemoresistance. Oncol Rep.2019;42:1035-

Page $12 / 18$ 


\section{Tables}

Table 1. Patients' characteristics

\begin{tabular}{|c|c|c|c|c|c|}
\hline Patient ID & Gender & Age (years) & $\begin{array}{c}\text { Leukemic blast cell } \\
\text { counts } \square \% \square\end{array}$ & FAB classification & MSCs Primary culture time (days) \\
\hline AML1 & Male & 47 & 12.8 & M3 & 22 \\
\hline AML2 & Female & 70 & 46.8 & M5 & 37 \\
\hline AML3 & Female & 18 & 13.3 & M2 & 23 \\
\hline AML4 & Male & 52 & 48.4 & M3 & 30 \\
\hline AML5 & Female & 38 & 20.7 & M3 & 28 \\
\hline AML6 & Male & 50 & 76 & M2 & 35 \\
\hline
\end{tabular}

Table 2. Influence of each MSC type on THP-1 cell viability, apoptosis, migration, and drug resistance

\begin{tabular}{ccccc}
\hline Items & $\begin{array}{c}\text { THP-1 cultured } \\
\text { alone }\end{array}$ & $\begin{array}{c}\text { Co-culture with HD- } \\
\text { MSC }\end{array}$ & $\begin{array}{c}\text { Co-culture with AML- } \\
\text { MSC }\end{array}$ & Co-culture with LCtrHD-MSCs \\
\hline $\begin{array}{c}\text { Cell viability } \\
(\%)\end{array}$ & 100 & $99.12 \pm 16.39$ & $115.77 \pm 13.26$ & $110.82 \pm 33.04$ \\
$\begin{array}{c}\text { Apoptosis } \\
(\%)\end{array}$ & 9.181 .23 & $7.02 \pm 1.29$ & $3.35 \pm 1.11$ & $4.32 \pm 1.42$ \\
$\begin{array}{c}\text { Cell migration } \\
\quad\left(\times 10^{5}\right)\end{array}$ & - & $0.66 \pm 0.25$ & $2.76 \pm 0.47$ & $1.92 \pm 0.26$ \\
$\begin{array}{c}\text { Chemoresistance } \\
(\%)\end{array}$ & $45.64 \pm 6.82$ & $34.99 \pm 3.61$ & $67.41 \pm 5.82$ & $59.49 \pm 11.00$ \\
\hline
\end{tabular}

\section{Supplemental Information Note}

Supplementary Figure. 1. Phenotype of cultured MSCs. Both HD- and AML-MSCs expressed typical MSC markers $\left(\mathrm{CD} 105^{+} \mathrm{CD} 29^{+} \mathrm{CD} 44^{+}\right)$and were negative for hematopoietic markers (CD 45 CD34- HLA-DR).

Supplementary Figure. 2. IC50 of Arc-a for MSCs and THP-1. MSCs at 24 h (A), 48 h (B), and THP-1 at 24 h (C), 48 h (D).

\section{Figures}



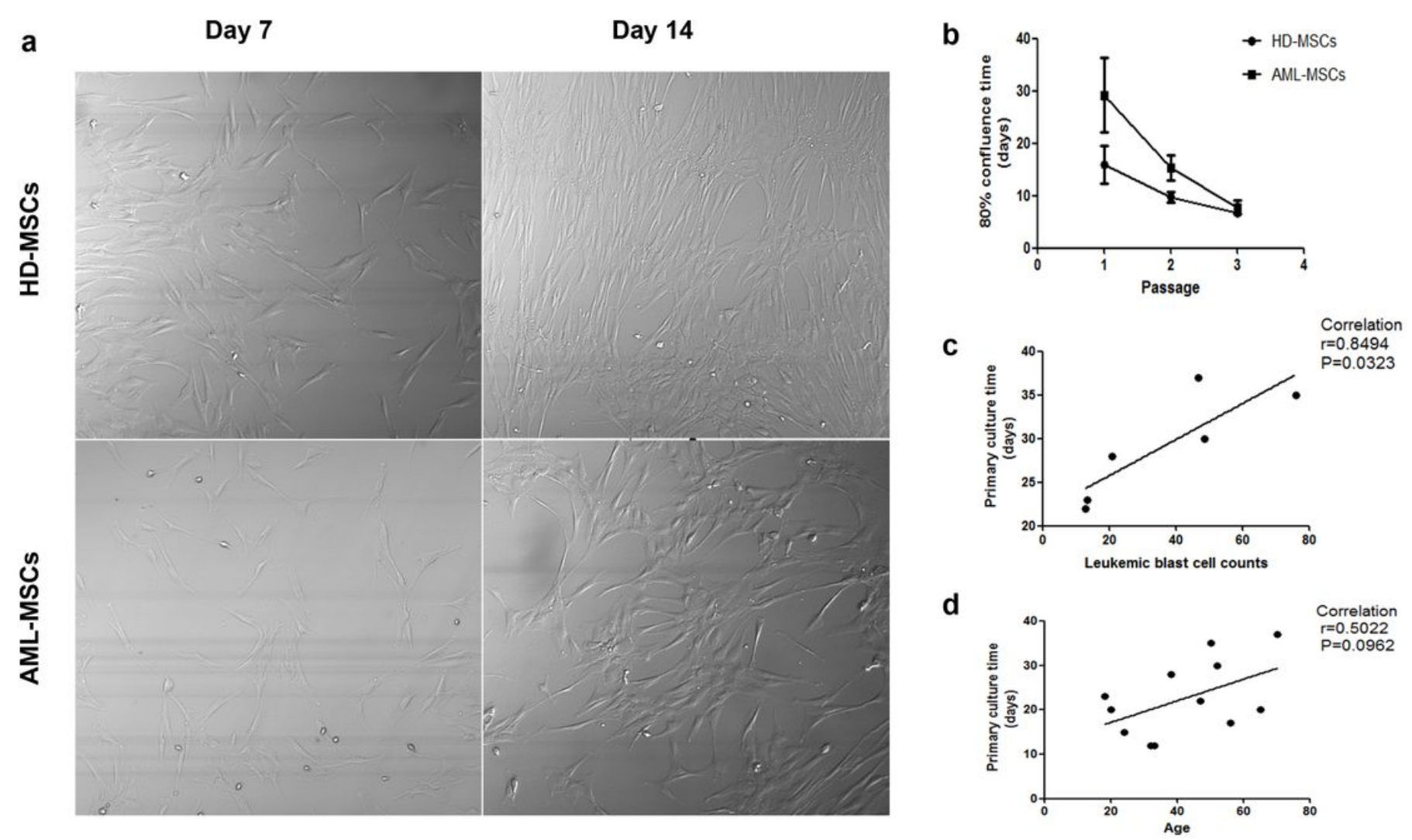

\section{Figure 1}

AML-MSCs showed reduced proliferative capacity. (A) The adherent cell numbers of AML-MSCs were significantly lower than HD-MSCs at day 7 and day 14 (×100); (B) Culture time (days) for passage 1, 2 and 3 of AML- and HD-MSCs. The AML-MSCs need more days to reach $80 \%$ confluency than HD-MSCs in passage 1 and 2, while in passage 3, the confluency time of the two MSCs was similar; (C) and (D) The correlation of primary culture time (confluency time of passage 1 ) with leukemic blast counts (C) and patient age (D). 

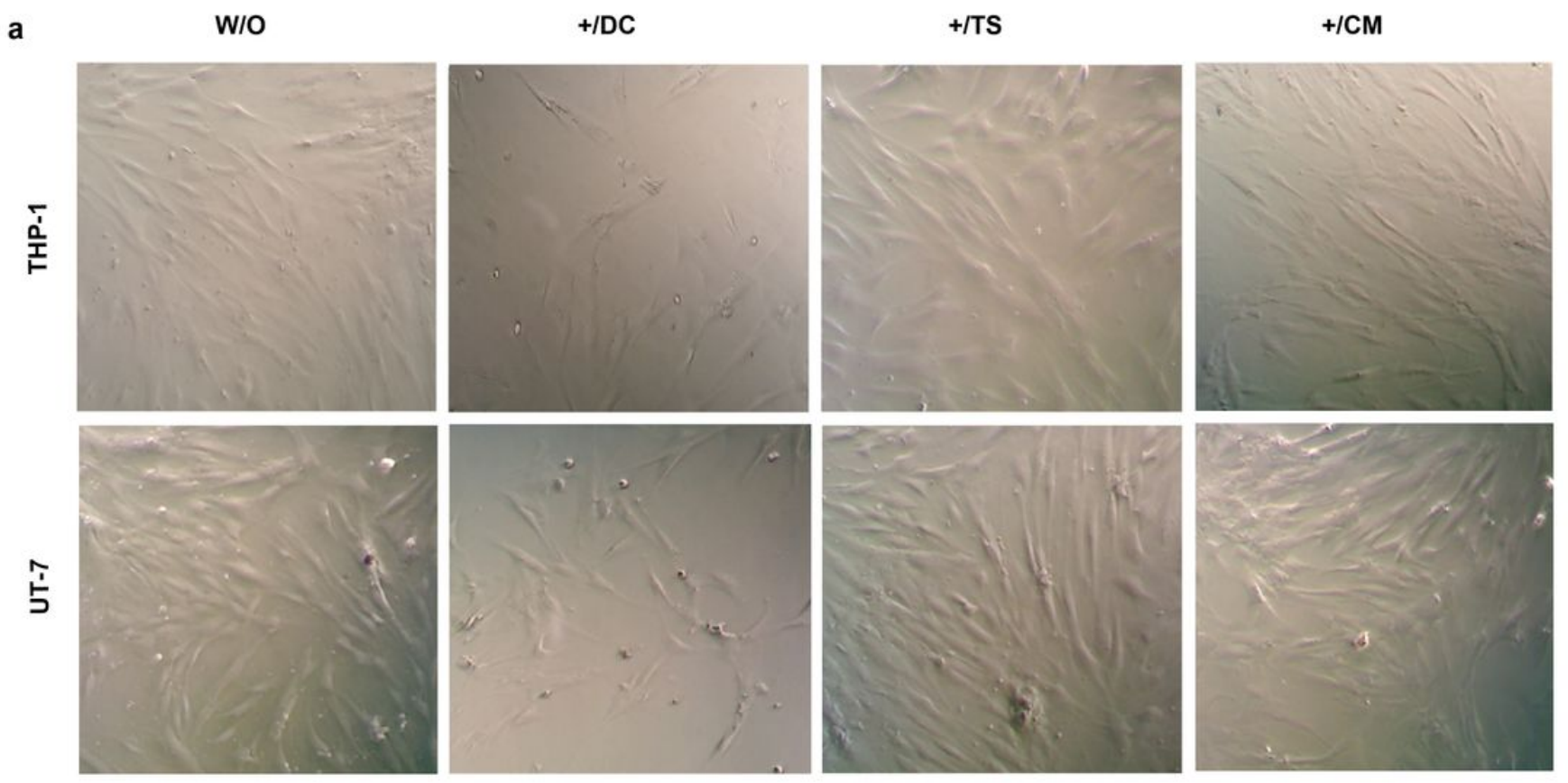

b
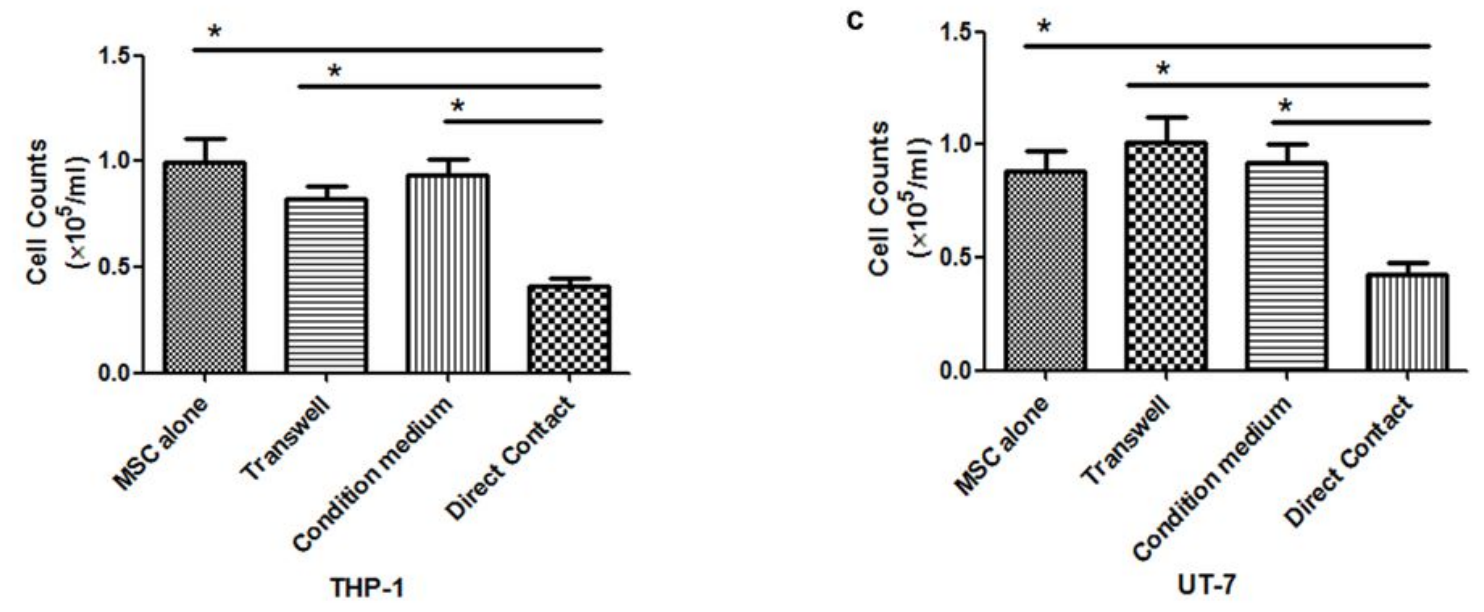

Figure 2

The influence of LCs on the proliferation of HD-MSCs. (A) Cell density and morphology of MSCs with or without co-culture of LCs. W/O, without LCs; +/DC, co-culture with LCs in direct connect way; +/TS, coculture with LCs in transwell system; $+/ C M$, culture with conditioned medium of LCs. (B) Cell numbers of MSCs after different culture conditions $(\times 105 / \mathrm{ml}),{ }^{*} p<0.05$. 
A

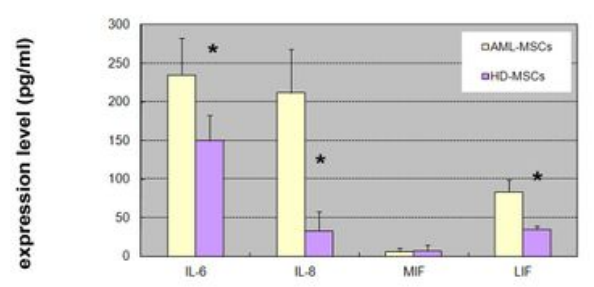

D

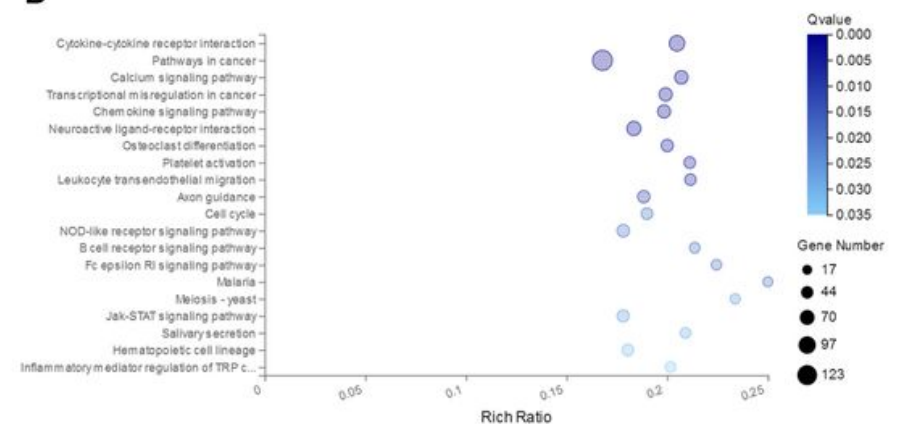

B

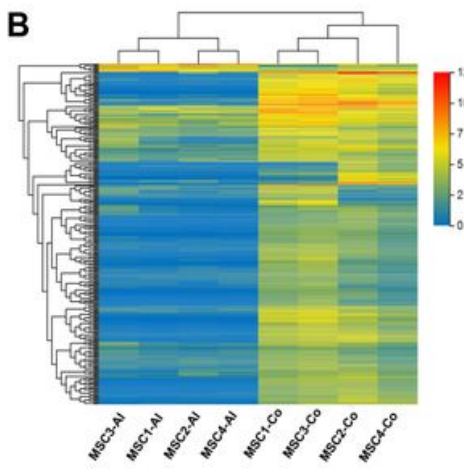

E

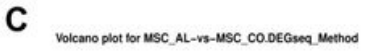

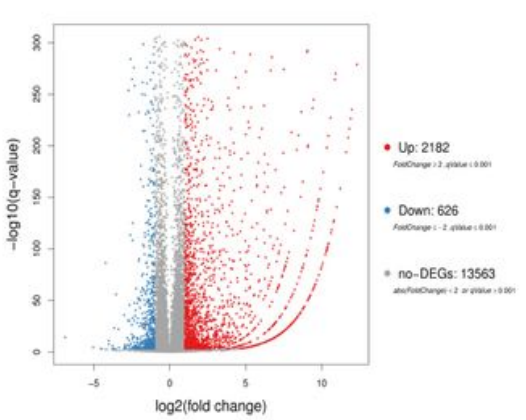

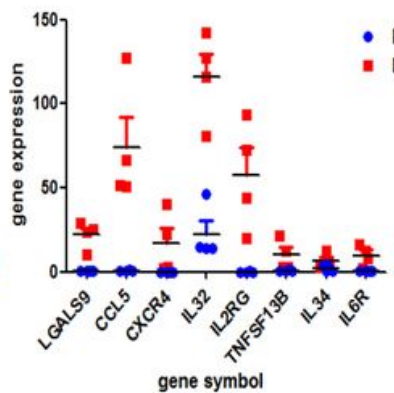

- MSC alone

- MSC co-culture

\section{Figure 3}

Influence of LCs on MSC gene expression and cytokine secretion. (A) Secretion of IL-6, IL-8 and MIF were elevated in AML-MSCs compared with HD-MSCs, * $p<0.05$. (B) Unsupervised hierarchical cluster analysis revealed different gene expression patterns between MSCs cultured alone (MSC-Al) and MSCs co-cultured with LCs (MSC-Co); (C) Differentially expressed genes between MSC-Al and MSC-Co (fold change $\geq 2$ or $\leq-2$ and $q$ value $\leq 0.001)$; (D) GO analysis of the differentially expressed genes; (E) Up-regulated expression of chemokines/inflammatory cytokines and their receptors in LCtrHD MSCs. 

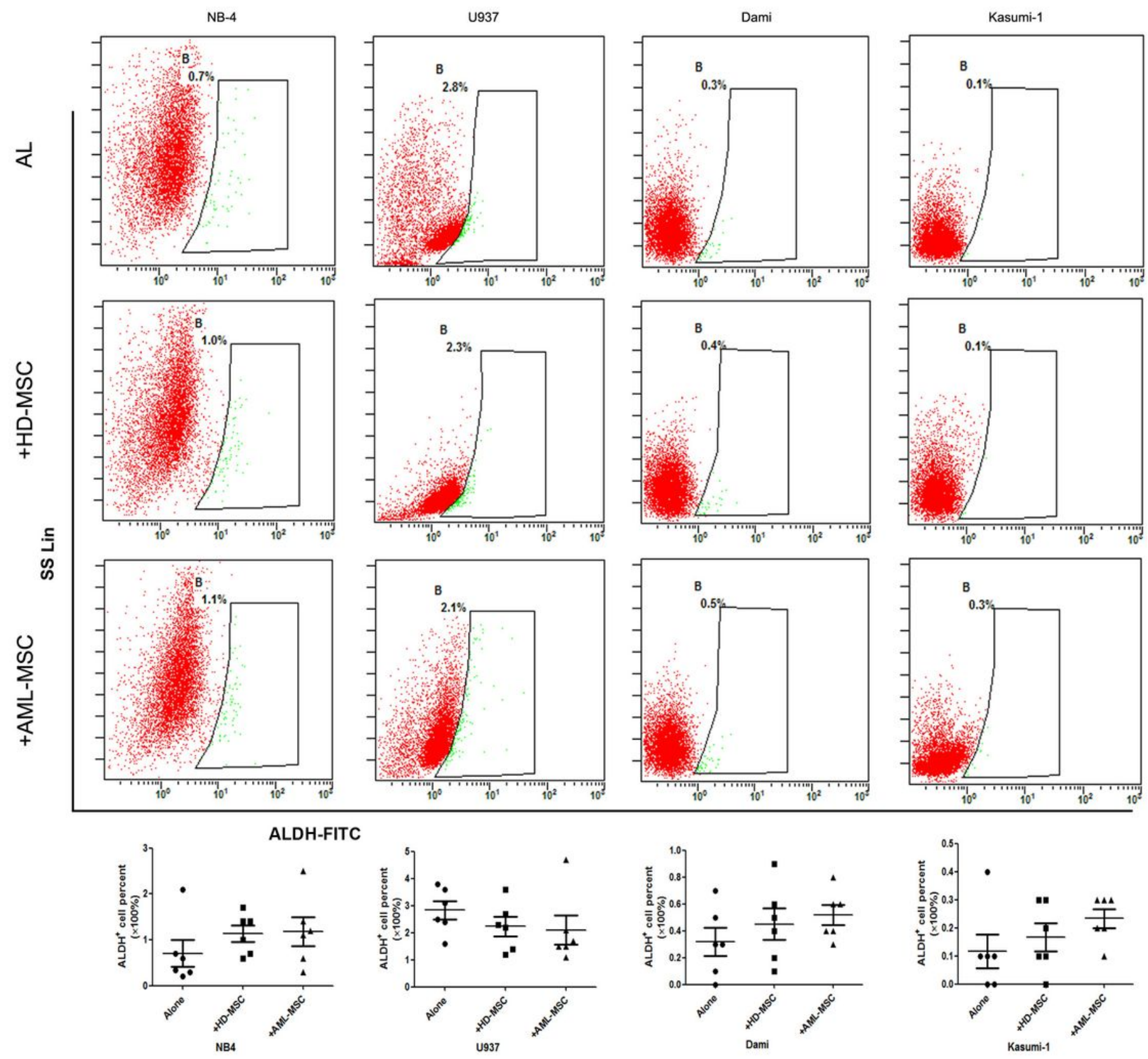

Figure 4

Percent ALDH+ cells in LC cultures. ALs, LCs cultured alone; +HD-MSCs, LCs co-cultured with HD-MSCs; +AML-MSCs, LCs co-cultured with AML-MSCs; $p>0.05$. 


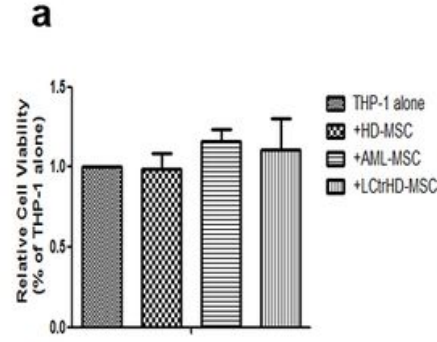

b

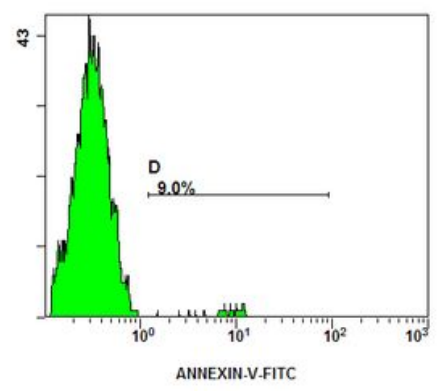

THP-1 alone c
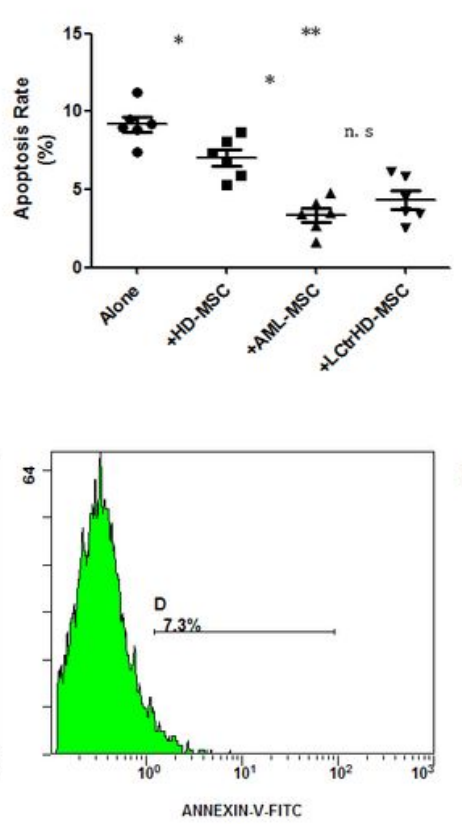

+HD-MSC

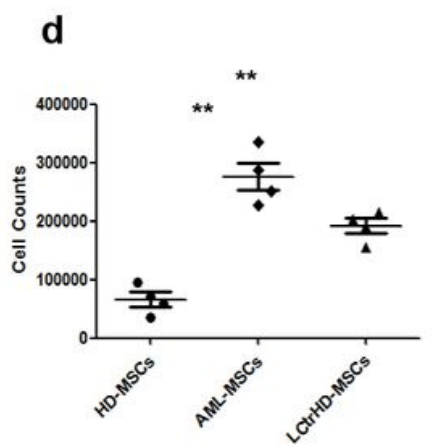

e
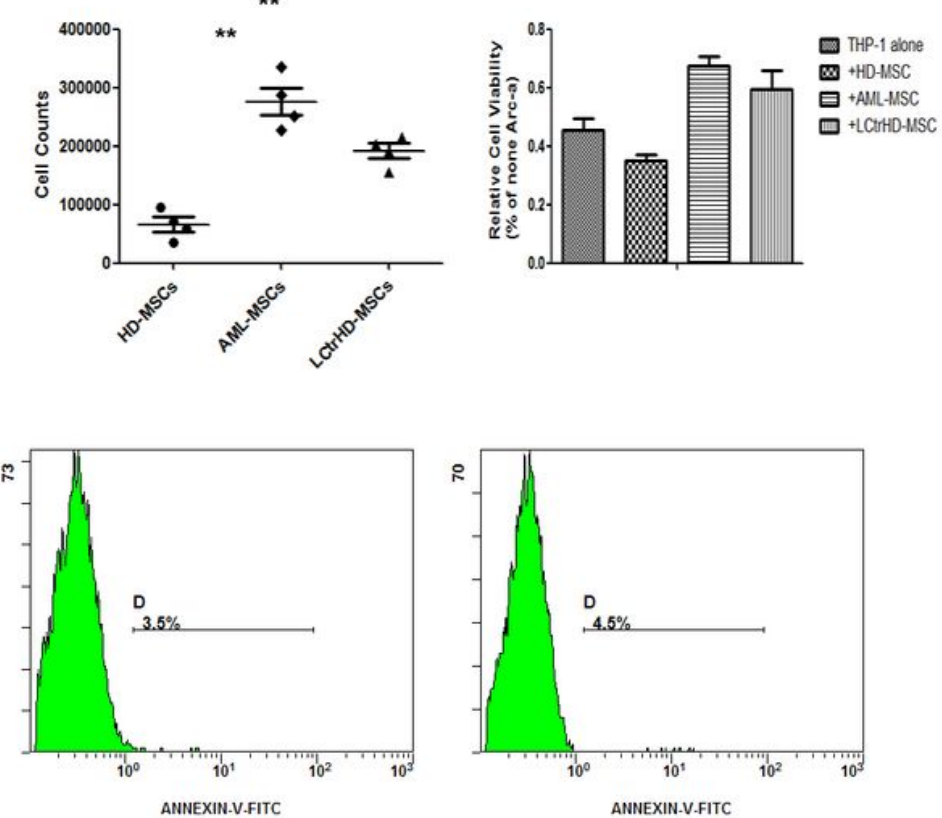

+AML-MSC

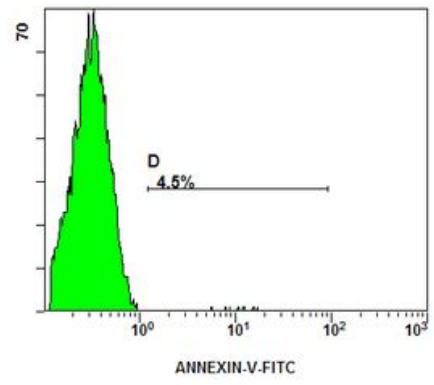

+LCtrHD-MSC

\section{Figure 5}

Biological function of different MSCs. THP-1 cells were cultured alone or co-cultured with HD-, AML-, or LCtrHD-MSCs (10:1). (A) Cell viability was examined using CCK-8 assay after $48 \mathrm{~h}$ in culture, and the relative cell viability was obtained by comparison with the viability of THP-1 cells cultured alone; (B) the apoptosis rate was estimated using flow cytometry; (C) THP-1 cell migration toward different MSCs; (D) Arc-a resistance of THP-1 cells in different culture conditions. THP-1 cells were cultured in the abovementioned culture conditions for $24 \mathrm{~h}, 1.2 \mathrm{mM}$ Arc-a was added to the culture system for $48 \mathrm{~h}$ and the cell viability was measured using the CCK-8 assay. ${ }^{*} p<0.05, * \star p<0.01, n . s$, not significant.

\section{Supplementary Files}

This is a list of supplementary files associated with this preprint. Click to download.

- SupplymentaryFig1.jpg

- SupplymentaryFig2.jpg 